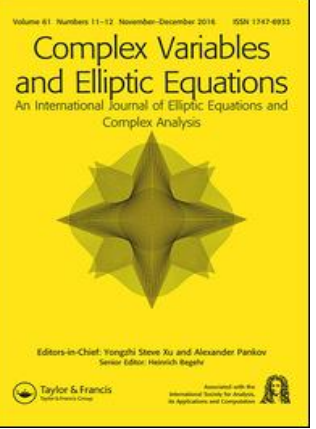

Complex Variables and Elliptic Equations

An International Journal

ISSN: 1747-6933 (Print) 1747-6941 (Online) Journal homepage: https://www.tandfonline.com/loi/gcov20

\title{
Some characterizations of magnetic Sobolev spaces
}

\section{Hoai-Minh Nguyen, Andrea Pinamonti, Marco Squassina \& Eugenio Vecchi}

To cite this article: Hoai-Minh Nguyen, Andrea Pinamonti, Marco Squassina \& Eugenio Vecchi (2018): Some characterizations of magnetic Sobolev spaces, Complex Variables and Elliptic Equations, DOI: 10.1080/17476933.2018.1520850

To link to this article: https://doi.org/10.1080/17476933.2018.1520850

Published online: 03 Oct 2018.

Submit your article to this journal

Џ Article views: 38

View Crossmark data $₫$ 


\title{
Some characterizations of magnetic Sobolev spaces
}

\author{
Hoai-Minh Nguyen ${ }^{a}$, Andrea Pinamonti ${ }^{b}$, Marco Squassina ${ }^{c}$ and Eugenio Vecchi ${ }^{d}$ \\ a Department of Mathematics, EPFL SB CAMA, Lausanne, Switzerland; ${ }^{\mathrm{b}}$ Dipartimento di Matematica, \\ Università di Trento, Povo (Trento), Italy; ${ }^{C}$ Dipartimento di Matematica e Fisica, Università Cattolica del Sacro

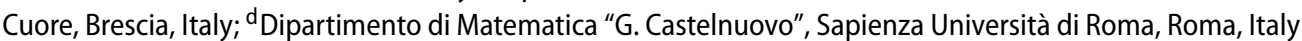

\begin{abstract}
The aim of this note is to survey recent results contained in Nguyen H-M, Squassina M. [On anisotropic Sobolev spaces. Commun Contemp Math, to appear. DOI:10.1142/S0219199718500177]; Nguyen $\mathrm{H}-\mathrm{M}$, Pinamonti A, Squassina $\mathrm{M}$, et al. [New characterizations of magnetic Sobolev spaces. Adv Nonlinear Anal. 2018;7(2):227-245]; Pinamonti A, Squassina M, Vecchi E. [Magnetic BV functions and the Bourgain-Brezis-Mironescu formula. Adv Calc Var, to appear. DOI:10.1515/acv-2017-0019]; Pinamonti A, Squassina M, Vecchi E. [The Maz'ya-Shaposhnikova limit in the magnetic setting.J Math Anal Appl. 2017;449:1152-1159] and Squassina M, Volzone B. [BourgainBrezis-Mironescu formula for magnetic operators. C R Math Acad Sci Paris. 2016;354:825-831], where the authors extended to the magnetic setting several characterizations of Sobolev and BV functions.
\end{abstract}

\section{ARTICLE HISTORY}

Received 2 June 2018

Accepted 30 August 2018

\section{COMMUNICATED BY}

D. Repovš

\section{KEYWORDS}

Magnetic Sobolev spaces; new characterization; nonlocal functionals

\section{AMS SUBJECT} CLASSIFICATIONS 49A50; 26A33; 82D99

\section{Introduction}

Let $\left\{\rho_{n}\right\}_{n \in \mathbb{N}}$ be a sequence of radial mollifiers, i.e. $\rho_{n}(x)=\rho_{n}(|x|)$, such that

$$
\begin{aligned}
\rho_{n} & \geq 0, \quad \int_{0}^{\infty} \rho_{n}(r) r^{N-1} \mathrm{~d} x=1, \quad \text { and } \\
\lim _{n \rightarrow \infty} \int_{\delta}^{\infty} \rho_{n}(r) r^{N-1} \mathrm{~d} r & =0 \quad \text { for every } \delta>0 .
\end{aligned}
$$

Let $\Omega$ be a smooth bounded open subset of $\mathbb{R}^{N}$ and let $p \geq 1$. In [1], Bourgain, Brézis, and Mironescu proved that, if $u \in L^{p}(\Omega)$ and

$$
\sup _{n \in \mathbb{N}} \int_{\Omega} \int_{\Omega} \frac{|u(x)-u(y)|^{p}}{|x-y|^{p}} \rho_{n}(|x-y|) \mathrm{d} x \mathrm{~d} y \leq C,
$$

for some positive constant $C$, then $u \in W^{1, p}(\Omega)$ if $p>1$ and $u \in B V(\Omega)$ if $p=1$. Moreover, one has

$$
\lim _{n \rightarrow \infty} \int_{\Omega} \int_{\Omega} \frac{|u(x)-u(y)|^{p}}{|x-y|^{p}} \rho_{n}(|x-y|) \mathrm{d} x \mathrm{~d} y=p Q_{p, N} \int_{\Omega}|\nabla u|^{p} \mathrm{~d} x
$$


Here

$$
Q_{p, N}:=\frac{1}{p} \int_{\mathbb{S}^{N-1}}|\omega \cdot \sigma|^{p} \mathrm{~d} \sigma,
$$

where $\mathbb{S}^{N-1} \subset \mathbb{R}^{N}$ denotes the unit sphere and $\boldsymbol{\omega}$ stands for an arbitrary unit vector of $\mathbb{R}^{N}$. Assertion (1) is established by Bourgain, Brézis, and Mironescu in [1] for $u \in W^{1, p}(\Omega)$ with $p \geq 1$. Assertion (1) with $p=1$ and $u \in B V(\Omega)$ is obtained by Davila [2]. In particular, we have the following celebrated Bourgain-Brézis-Mironescu (BBM) formula, for every $u \in W^{1, p}(\Omega)$,

$$
\lim _{s \rightarrow 1^{-}}(1-s) \int_{\Omega} \int_{\Omega} \frac{|u(x)-u(y)|^{p}}{|x-y|^{N+s p}} \mathrm{~d} x \mathrm{~d} y=Q_{p, N} \int_{\Omega}|\nabla u|^{p} \mathrm{~d} x .
$$

Other properties related to the BBM formula can be found in [3-5]. In the spirit of (3), Maz'ya and Shaposhnikova proved in [6] that for any $p \in[1, \infty)$,

$$
\lim _{s \searrow 0} s \int_{\mathbb{R}^{N}} \int_{\mathbb{R}^{N}} \frac{|u(x)-u(y)|^{p}}{|x-y|^{N+p s}} \mathrm{~d} x \mathrm{~d} y=\frac{4 \pi^{N / 2}}{p \Gamma(N / 2)}\|u\|_{L^{p}\left(\mathbb{R}^{N}\right)}^{p},
$$

whenever $u \in D_{0}^{s, p}\left(\mathbb{R}^{N}\right)$ for some $s \in(0,1)$. Here $\Gamma$ denotes the Gamma function and the space $D_{0}^{s, p}\left(\mathbb{R}^{N}\right)$ is the completion of $C_{c}^{\infty}\left(\mathbb{R}^{N}\right)$ with respect to the Gagliardo semi-norm.

Other characterizations of Sobolev spaces and BV functions which are somewhat related to the one of Bourgain, Brézis, and Mironescu are established in $[7,8]$. For example, in the case $p=2$, the following characterization of $H^{1}(\Omega)$ is given in $[7,8]$. Set

$$
I_{\delta}(u):=\iint_{\substack{\Omega \times \Omega \\\{|u(y)-u(x)|>\delta\}}} \frac{\delta^{2}}{|x-y|^{N+2}} \mathrm{~d} x \mathrm{~d} y, \quad \text { for } u \in L_{\text {loc }}^{1}(\Omega) \text { and } \delta>0 .
$$

Then for any $u \in L^{2}(\Omega), u \in H^{1}(\Omega)$ if and only if $\sup _{0<\delta<1} I_{\delta}(u)<\infty$. Moreover, for every $u \in H^{1}(\Omega)$

$$
\lim _{\delta \searrow 0} I_{\delta}(u)=Q_{2, N} \int_{\Omega}|\nabla u|^{2} \mathrm{~d} x
$$

where $Q_{2, N}$ is the same positive constant appearing in (3) for $p=2$. Other results related to the nonlocal operator $I_{\delta}$ can be found in [9-13]. The aim of this note is to survey recent results contained in [14-18], where the authors have extended the aforementioned results to the magnetic setting. We refer the interested reader to these papers for the proofs and further details.

\section{Magnetic Sobolev and BV spaces}

An important role in the study of particles which interact with a magnetic field $B=\nabla \times A$, $A: \mathbb{R}^{3} \rightarrow \mathbb{R}^{3}$, is played by an extension of the Laplacian, known as magnetic Laplacian $(\nabla-\mathrm{i} A)^{2}$ (see $\left.[19,20]\right)$. Nonlinear magnetic Schrödinger equations like

$$
-(\nabla-\mathrm{i} A)^{2} u+u=f(u)
$$

have been extensively studied (see e.g. [19,21-23] and the references therein). The functional framework to work with these equations is the magnetic Sobolev spaces which will 
be now recalled, see [24] for a concise introduction to the topic. For $p \geq 1$, let us endow the vector space $\mathbb{C}^{N}$ with the norm

$$
|z|_{p}:=\left(\left|\left(\Re z_{1}, \ldots, \Re z_{N}\right)\right|^{p}+\left|\left(\Im z_{1}, \ldots, \Im z_{N}\right)\right|^{p}\right)^{1 / p},
$$

where $\Re a$, $\Im a$ denote the real and imaginary parts of $a \in \mathbb{C}$ respectively, and $|\cdot|$ the Euclidean norm of $\mathbb{R}^{N}$. We notice that $|z|_{p}=|z|$ whenever $z \in \mathbb{R}^{N}$. We warn the reader that in the non Hilbert case $p \neq 2$, this choice for a norm on $\mathbb{C}^{N}$ is different from the standard one. Continuing with the notation, we will denote the imaginary unit by $i$, and we denote by $L^{p}(\Omega, \mathbb{C})$ the Lebesgue space naturally associated to $|\cdot|_{p}$.

We are ready to introduce

Definition 2.1: Let $p \geq 1$ and $A: \mathbb{R}^{N} \rightarrow \mathbb{R}^{N}$ be a measurable function. The magnetic Sobolev space $W_{A}^{1, p}(\Omega)$ is given by

$$
W_{A}^{1, p}(\Omega):=\left\{u \in L^{p}(\Omega, \mathbb{C}):[u]_{W_{A}^{1, p}(\Omega)}<\infty\right\},
$$

where

$$
[u]_{W_{A}^{1, p}(\Omega)}:=\left(\int_{\Omega}|\nabla u-\mathrm{i} A(x) u|_{p}^{p} \mathrm{~d} x\right)^{1 / p} .
$$

The space $W_{A}^{1, p}(\Omega)$ is equipped with the following norm

$$
\|u\|_{W_{A}^{1, p}(\Omega)}:=\left(\|u\|_{L^{p}(\Omega)}^{p}+[u]_{W_{A}^{1, p}(\Omega)}^{p}\right)^{1 / p} .
$$

We can also define the space $W_{0, A}^{1, p}(\Omega)$ as the closure of $C_{c}^{\infty}(\Omega)$ in $W_{A}^{1, p}(\Omega)$. As a notational remark, as it is customary, when $p=2$ we will denote the magnetic Sobolev space $W_{A}^{1,2}(\Omega)$ by $H_{A}^{1}(\Omega)$.

A possibility to define a suitable notion of fractional magnetic Sobolev space is to use the energy space of a non-local operator on $\mathbb{R}^{N}$, see $[25,26]$. There are at least three possible notions of magnetic fractional Laplacian which are in general not equivalent, see the survey of Ichinose in [26]. The most frequently used operator is $(-\Delta)_{A}^{s}$, which is defined as the gradient of the non-local energy functional

$$
u \mapsto \iint_{\mathbb{R}^{2 N}} \frac{\left|u(x)-\mathrm{e}^{\mathrm{i}(x-y) \cdot A((x+y) / 2)} u(y)\right|^{2}}{|x-y|^{N+2 s}} \mathrm{~d} x \mathrm{~d} y,
$$

namely

$$
(-\Delta)_{A}^{s} u(x)=c(N, s) \lim _{\varepsilon \searrow 0} \int_{B_{\varepsilon}^{c}(x)} \frac{u(x)-\mathrm{e}^{\mathrm{i}(x-y) \cdot A((x+y) / 2)} u(y)}{|x-y|^{N+2 s}} \mathrm{~d} y,
$$

where

$$
\lim _{s \nearrow 1} \frac{c(N, s)}{1-s}=\frac{4 N \Gamma(N / 2)}{2 \pi^{N / 2}} .
$$

Recently, the operator $(-\Delta)_{A}^{s}$ has been investigated in several directions. Here is a brief (and far from being complete) list of references: [27-33] 
We are ready to introduce the non-local counterpart of the magnetic Sobolev spaces:

Definition 2.2: Let $A: \mathbb{R}^{N} \rightarrow \mathbb{R}^{N}$ be a locally bounded measurable function and let $\Omega \subset$ $\mathbb{R}^{N}$ be an open set. For any $s \in(0,1)$ and $p \geq 1$, the magnetic Gagliardo semi-norm is defined as

$$
[u]_{W_{A}^{s, p}(\Omega)}:=\left(\int_{\Omega} \int_{\Omega} \frac{\left|u(x)-\mathrm{e}^{\mathrm{i}(x-y) \cdot A((x+y) / 2)} u(y)\right|_{p}^{p}}{|x-y|^{N+p s}} \mathrm{~d} x \mathrm{~d} y\right)^{1 / p} .
$$

The fractional magnetic Sobolev space $W_{A}^{s, p}(\Omega)$ is given by

$$
W_{A}^{s, p}(\Omega):=\left\{u \in L^{p}(\Omega, \mathbb{C}):[u]_{W_{A}^{s, p}(\Omega)}<\infty\right\}
$$

and it is equipped with the norm

$$
\|u\|_{W_{A}^{s, p}(\Omega)}:=\left(\|u\|_{L^{p}(\Omega)}^{p}+[u]_{W_{A}^{s, p}(\Omega)}^{p}\right)^{1 / p} .
$$

We stress that for $A \equiv 0$ and $u$ real-valued, the above definition is consistent with the usual fractional Sobolev space $W^{s, p}(\Omega)$ endowed with the classical norm

$$
\|\cdot\|_{W^{s, p}(\Omega)}=\left(\|u\|_{L^{p}(\Omega)}^{p}+\int_{\Omega} \int_{\Omega} \frac{|u(x)-u(y)|_{p}^{p}}{|x-y|^{N+p s}} \mathrm{~d} x \mathrm{~d} y\right)^{1 / p} .
$$

Remark 2.1: As it is pointed out in [26], in place of the magnetic norm defined via the simple midpoint prescription $(x, y) \mapsto A((x+y) / 2)$, other prescriptions are viable in applications such as the averaged one

$$
(x, y) \mapsto \int_{0}^{1} A((1-\vartheta) x+\vartheta y) d \vartheta=: A_{\sharp}(x, y) .
$$

If $(-\Delta)_{A}^{s}$ and $(-\Delta)_{A_{\sharp}}^{s}$ are the fractional operators associated with $A((x+y) / 2)$ and $A_{\sharp}(x, y)$ respectively, it follows that $(-\Delta)_{A_{\sharp}}^{s}$ is Gauge covariant, which is relevant for Schrödinger operators, i.e. for all $\phi \in \mathscr{S}\left(\mathbb{R}^{n}\right)$

$$
(-\Delta)_{(A+\nabla \phi)_{\sharp}}^{S}=\mathrm{e}^{\mathrm{i} \phi}(-\Delta)_{A_{\sharp}}^{s} \mathrm{e}^{-\mathrm{i} \phi},
$$

see e.g. [26, Proposition 2.8].

We present now the notion of magnetic bounded variation functions introduced in [16].

Definition 2.3 ( $A$-bounded variation functions): Let $\Omega \subset \mathbb{R}^{N}$ be an open set and let $A: \mathbb{R}^{N} \rightarrow \mathbb{R}^{N}$ be a locally bounded function. A function $u \in L^{1}(\Omega, \mathbb{C})$ is said to be of 
$A$-bounded variation and we write $u \in B V_{A}(\Omega)$, if

$$
|D u|_{A}(\Omega):=C_{1, A, u}(\Omega)+C_{2, A, u}(\Omega)<\infty
$$

where we set

$$
\begin{aligned}
C_{1, A, u}(\Omega) & :=\sup \left\{\int_{\Omega} \Re u(x) \operatorname{div} \varphi(x)-A(x) \cdot \varphi(x) \Im u(x) \mathrm{d} x \mid \varphi\right. \\
& \left.\in C_{c}^{\infty}\left(\Omega, \mathbb{R}^{N}\right),\|\varphi\|_{L^{\infty}(\Omega)} \leq 1\right\}, \\
C_{2, A, u}(\Omega) & :=\sup \left\{\int_{\Omega} \Im u(x) \operatorname{div} \varphi(x)+A(x) \cdot \varphi(x) \Re u(x) \mathrm{d} x \mid \varphi\right. \\
& \left.\in C_{c}^{\infty}\left(\Omega, \mathbb{R}^{N}\right),\|\varphi\|_{L^{\infty}(\Omega)} \leq 1\right\} .
\end{aligned}
$$

A function $u \in L_{\text {loc }}^{1}(\Omega, \mathbb{C})$ is said to be of locally $A$-bounded variation and we write $u \in$ $B V_{A, l o c}(\Omega)$, if

$$
|D u|_{A}(U)<\infty, \quad \text { for every open set } U \Subset \Omega \text {. }
$$

We endow the space $B V_{A}(\Omega, \mathbb{C})$ with the following norm:

$$
\|u\|_{B V_{A}(\Omega)}:=\|u\|_{L^{1}(\Omega)}+|D u|_{A}(\Omega) .
$$

With this choice, the space $\left(B V_{A}(\Omega),\|\cdot\|_{B V_{A}(\Omega)}\right)$ is a real Banach space, see [16, Lemma 3.8].

As for the magnetic Sobolev spaces, in the case $A \equiv 0$, the previous definition is consistent with the classical one of $B V(\Omega)$. We summarize now the basic properties of the space $B V_{A}(\Omega)$ that has been fully proved in [16]. The coming results can be considered as the natural extension to the magnetic setting of the classical theory, see e.g. [34].

Lemma 2.1 ([16, Lemma 3.2]): Let $\Omega \subset \mathbb{R}^{N}$ be an open and bounded set, $A: \mathbb{R}^{N} \rightarrow \mathbb{R}^{N}$ locally bounded and $u \in B V_{A}(\Omega)$. Let $E \subset \Omega$ be a Borel set then

$$
\begin{aligned}
|D u|_{A}(E):= & \inf \left\{C_{1, A, u}(U) \mid E \subset U, U \subset \Omega \text { open }\right\} \\
& +\inf \left\{C_{2, A, u}(U) \mid E \subset U, U \subset \Omega \text { open }\right\}
\end{aligned}
$$

extends $|D u|_{A}(\cdot)$ to a Radon measure in $\Omega$. For any open set $U \subset \Omega, C_{1, A, u}(U)$ and $C_{2, A, u}(U)$ are defined requiring the test functions to be supported in $U$ and $|D u|_{A}(\emptyset):=0$.

Lemma 2.2 ([16, Lemma 3.3]): Let $\Omega \subset \mathbb{R}^{N}$ be an open set. Let $A: \mathbb{R}^{N} \rightarrow \mathbb{R}^{N}$ be locally bounded. Then

$$
W_{\mathrm{loc}}^{1,1}(\Omega) \subset B V_{A, \mathrm{loc}}(\Omega)
$$


Lemma 2.3 ([16, Lemma 3.4]): Let $\Omega \subset \mathbb{R}^{N}$ be an open set. Let $A: \mathbb{R}^{N} \rightarrow \mathbb{R}^{N}$ be locally bounded. Assume that $u \in W_{A}^{1,1}(\Omega)$. Then $u \in B V_{A}(\Omega)$ and it holds

$$
|D u|_{A}(\Omega)=\int_{\Omega}|\nabla u-\mathrm{i} A(x) u|_{1} \mathrm{~d} x
$$

Furthermore, if $u \in B V_{A}(\Omega) \cap C^{\infty}(\Omega)$, then $u \in W_{A}^{1,1}(\Omega)$.

Lemma 2.4 ([16, Lemma 3.5]): Let $\Omega \subset \mathbb{R}^{N}$ be an open and bounded set. Let $A: \mathbb{R}^{N} \rightarrow$ $\mathbb{R}^{N}$ be locally bounded. Then $u \in B V_{A}(\Omega)$ if and only if $u \in B V(\Omega)$. Moreover, for every $u \in B V_{A}(\Omega)$, there exists a positive constant $K=K(A, \Omega)$ such that

$$
K^{-1}\|u\|_{B V(\Omega)} \leq\|u\|_{B V_{A}(\Omega)} \leq K\|u\|_{B V(\Omega)} .
$$

Lemma 2.5 ([16, Lemma 3.7]): Let $A: \mathbb{R}^{N} \rightarrow \mathbb{R}^{N}$ be locally bounded. Let $\Omega \subset \mathbb{R}^{N}$ be an open set and $\left\{u_{k}\right\}_{k \in \mathbb{N}} \subset B V_{A}(\Omega)$ a sequence converging locally in $L^{1}(\Omega)$ to a function $u$. Then

$$
\liminf _{k \rightarrow \infty}\left|D u_{k}\right|_{A}(\Omega) \geq|D u|_{A}(\Omega)
$$

Lemma 2.6 ([16, Lemma 3.10]): Suppose that $A: \mathbb{R}^{N} \rightarrow \mathbb{R}^{N}$ is locally Lipschitz. Let $\Omega \subset$ $\mathbb{R}^{N}$ be an open and bounded set and let $u \in B V_{A}(\Omega)$. Then there exists a sequence $\left\{u_{k}\right\}_{k \in \mathbb{N}} \subset$ $C^{\infty}(\Omega, \mathbb{C})$ such that

$$
\lim _{k \rightarrow \infty} \int_{\Omega}\left|u_{k}-u\right|_{1} \mathrm{~d} x=0 \quad \text { and } \quad \lim _{k \rightarrow \infty}\left|D u_{k}\right|_{A}(\Omega)=|D u|_{A}(\Omega)
$$

Lemma 2.7 ([16, Lemma 3.14]): Assume that $\Omega \subset \mathbb{R}^{N}$ is a bounded domain with Lipschitz boundary and that $A: \mathbb{R}^{N} \rightarrow \mathbb{R}^{N}$ is locally bounded. Let $\left\{u_{k}\right\}_{k \in \mathbb{N}}$ be a bounded sequence in $B V_{A}(\Omega)$. Then, up to a subsequence, it converges strongly in $L^{1}(\Omega)$ to some function $u \in$ $B V_{A}(\Omega)$.

Lemma 2.8 ([16, Lemma 3.12]): Let $\Omega \subset \mathbb{R}^{N}$ be an open bounded set with Lipschitz boundary and let $A: \mathbb{R}^{N} \rightarrow \mathbb{R}^{N}$ be locally Lipschitz. Then for any open set $W \supset \bar{\Omega}$, there exists a linear and continuous extension operator $E: B V_{A}(\Omega) \rightarrow B V_{A}\left(\mathbb{R}^{N}\right)$ such that

$$
E u=0, \quad \text { for almost every } x \in \mathbb{R}^{N} \backslash W, \quad \text { and }|D E u|_{A}(\partial \Omega)=0 \text {, }
$$

for every $u \in B V_{A}(\Omega)$.

A few words concerning the proofs of the aforementioned results are now in order. Roughly speaking, the strategy of the proofs follow the classical ones as in e.g. [34]. From the technical point of view, once we ask for local boundedness of $A$ we can usually control the extra-terms coming from $A$. In particular, the norm equivalence provided by Lemma 2.4 and the pointwise Diamagnetic inequality, see e.g. [28] allow sometimes to get magnetic results from the classical ones. We refer to [16, Section 3] for more details. 


\section{Magnetic BBM-type formulas}

The introduction of the magnetic counterpart of classical Sobolev spaces and BV space leads to the following natural question: do BBM-type formulas still hold in the magnetic setting? The aim of this section is to collect some results that provide a positive answer to the above question.

An useful equality to get BBM-type formulas is

$$
\int_{\mathbb{S}^{N-1}}|v \cdot \sigma|_{p}^{p} \mathrm{~d} \sigma=p Q_{p, N}|v|_{p}^{p}, \quad \text { for all } v \in \mathbb{C}^{N}, p \geq 1
$$

This motivates the introduction of the norm $|\cdot|_{p}$ on $\mathbb{C}^{N}$. Indeed, (4) does not hold with the classical Euclidean norm for $p \neq 2$. Given $u: \mathbb{R}^{N} \rightarrow \mathbb{C}$ a measurable complex-valued function, we denote

$$
\Psi_{u}(x, y):=\mathrm{e}^{\mathrm{i}(x-y) \cdot A((x+y) / 2)} u(y), \quad x, y \in \mathbb{R}^{N} .
$$

The function $\Psi_{u}(\cdot, \cdot)$ also depends on $A$ but for notational ease, we ignore it.

Theorem 3.1 (Magnetic Bourgain-Brezis-Mironescu type result): Let $p \geq 1, A: \bar{\Omega} \rightarrow$ $\mathbb{R}^{N}$ be of class $C^{1}$ and let $\left\{\rho_{n}\right\}_{n \in \mathbb{N}}$ be a sequence of nonnegative radial mollifiers. Then $u \in$ $W_{A}^{1, p}(\Omega)$ if $p>1$ and $u \in B V_{A}(\Omega)$ if $p=1$ if and only if $u \in L^{p}(\Omega)$ and

$$
\sup _{n \in \mathbb{N}} \int_{\Omega} \int_{\Omega} \frac{\left|\Psi_{u}(x, y)-\Psi_{u}(x, x)\right|_{p}^{p}}{|x-y|^{p}} \rho_{n}(|x-y|) \mathrm{d} x \mathrm{~d} y<+\infty .
$$

Moreover,

$$
\lim _{n \rightarrow+\infty} \int_{\Omega} \int_{\Omega} \frac{\left|\Psi_{u}(x, y)-\Psi_{u}(x, x)\right|_{p}^{p}}{|x-y|^{p}} \rho_{n}(|x-y|) \mathrm{d} x \mathrm{~d} y=Q_{1, N}|D u|_{A}(\Omega) .
$$

Statement (5) is proved in [18] for $p=2$, in [16] for $p=1$ both under the assumption that $A \in C^{2}(\bar{\Omega})$, in [15] for $p>1$ and $A \in C^{1}(\bar{\Omega})$, and for $p \geq 1$ for a more general (anisotropic) setting in [14]. The proof of (6) is given in [15] for $p>1$ and for $p \geq 1$ for a more general setting in [14]. ${ }^{1}$ The proof of Theorem 3.1 is essentially based on the works in the case without magnetic field, see $[1,2,35]$. Nevertheless work is required to deal with the presence of the magnetic field $A$.

\section{A magnetic version of the result by Maz'ya and Shaposhnikova}

The aim of this section is to describe the generalization proved in [17] of [6] to the magnetic case. For a locally bounded $A$, let the space of complex valued functions $D_{A, 0}^{s, p}\left(\mathbb{R}^{N}, \mathbb{C}\right)$ be the completion of $C_{c}^{\infty}\left(\mathbb{R}^{N}, \mathbb{C}\right)$ with respect to the norm

$$
\|u\|_{D_{A, 0}^{s, p}}=\left(\int_{\mathbb{R}^{N}} \int_{\mathbb{R}^{N}} \frac{\left|\Psi_{u}(x, y)-\Psi_{u}(x, x)\right|^{p}}{|x-y|^{N+p s}} \mathrm{~d} x \mathrm{~d} y\right)^{1 / p} .
$$


Theorem 4.1 (Magnetic Maz'ya-Shaposhnikova type result): Let $N \geq 1$ and $p \in[1, \infty)$. Then for every

$$
u \in \bigcup_{0<s<1} D_{A, 0}^{s, p}\left(\mathbb{R}^{N}, \mathbb{C}\right)
$$

there holds

$$
\lim _{s \searrow 0} s \int_{\mathbb{R}^{N}} \int_{\mathbb{R}^{N}} \frac{\left|\Psi_{u}(x, x)-\Psi_{u}(x, y)\right|^{p}}{|x-y|^{N+p s}} \mathrm{~d} x \mathrm{~d} y=\frac{4 \pi^{N / 2}}{p \Gamma(N / 2)}\|u\|_{L^{p}\left(\mathbb{R}^{N}\right)}^{p} .
$$

In one direction the proof is based on the Diamagnetic inequality to reduce the problem to the non-magnetic case. For the converse inequality, the magnetic effects has to be controlled, and this can be done because the magnetic effect becomes negligible as $s \rightarrow 0$.

Remark 4.1: We point out that when $A \equiv 0$ then Theorem 4.1 boils down to the result proved in [6]. It also remains valid for the operator $A_{\sharp}$ and its proof carries on by trivial modifications of our arguments.

\section{A magnetic version of the results by Bourgain and Nguyen}

In this section, we present some results in [15]. Set

$$
J_{\delta}(u):=\iint_{\left\{\left|\Psi_{u}(x, y)-\Psi_{u}(x, x)\right|>\delta\right\}} \frac{\delta^{2}}{|x-y|^{N+2}} \mathrm{~d} x \mathrm{~d} y, \quad \text { for } u \in L_{\text {loc }}^{1}\left(\mathbb{R}^{N}\right), \delta>0 \text {. }
$$

We prove

Theorem 5.1: Let $A: \mathbb{R}^{N} \rightarrow \mathbb{R}^{N}$ be Lipschitz. Then $u \in H_{A}^{1}\left(\mathbb{R}^{N}\right)$ if and only if $u \in L^{2}\left(\mathbb{R}^{N}\right)$ and

$$
\sup _{0<\delta<1} J_{\delta}(u)<+\infty
$$

Moreover, we have, for $u \in H_{A}^{1}\left(\mathbb{R}^{N}\right)$,

$$
\lim _{\delta \searrow 0} J_{\delta}(u)=Q_{N} \int_{\mathbb{R}^{N}}|\nabla u-\mathrm{i} A(x) u|^{2} \mathrm{~d} x
$$

and

$$
\sup _{\delta>0} J_{\delta}(u) \leq C_{N}\left(\int_{\mathbb{R}^{N}}|\nabla u-\mathrm{i} A(x) u|^{2} \mathrm{~d} x+\left(\|\nabla A\|_{L^{\infty}\left(\mathbb{R}^{N}\right)}^{2}+1\right) \int_{\mathbb{R}^{N}}|u|^{2} \mathrm{~d} x\right) .
$$

This provides a new characterization of the $H_{A}^{1}$ norm in terms of nonlocal functionals extending to the magnetic setting further results in the spirit of Bourgain, Brézis and Mironescu [1,36] (see also [2,37]). 


\section{Almost everywhere and $L^{1}$ convergence}

In this section we collect other results obtained in [15] in the spirit of the works [4,38]. We are there interested in other modes of convergence of functionals related to those appearing in Theorems 3.1 and 5.1. We only recall some results for the case $p=2$. For $u \in L_{\text {loc }}^{1}\left(\mathbb{R}^{N}\right)$, set

$$
D_{n}(u, x):=\int_{\mathbb{R}^{N}} \frac{\left|\Psi_{u}(x, y)-\Psi_{u}(x, x)\right|^{2}}{|x-y|^{2}} \rho_{n}(|x-y|) \mathrm{d} y, \quad \text { for } x \in \mathbb{R}^{N} .
$$

Concerning Theorem 3.1, we have

Proposition 6.1 ([15, Proposition 4.1]): Let $A: \mathbb{R}^{N} \rightarrow \mathbb{R}^{N}$ be Lipschitz, $u \in H_{A}^{1}\left(\mathbb{R}^{N}\right)$, and let $\left(\rho_{n}\right)$ be a sequence of radial mollifiers such that

$$
\sup _{t>1} \sup _{n} t^{-2} \rho_{n}(t)<+\infty \text {. }
$$

We have

$$
\lim _{n \rightarrow+\infty} D_{n}(u, x)=2 Q_{N}|\nabla u(x)-\mathrm{i} A(x) u(x)|^{2}, \quad \text { for a.e. } x \in \mathbb{R}^{N},
$$

and

$$
\lim _{n \rightarrow+\infty} D_{n}(u, \cdot)=2 Q_{N}|\nabla u(\cdot)-\mathrm{i} A(\cdot) u(\cdot)|^{2}, \quad \text { in } L^{1}\left(\mathbb{R}^{N}\right) .
$$

Concerning Theorem 5.1, we set, for $u \in L_{\text {loc }}^{1}\left(\mathbb{R}^{N}\right)$ and $x \in \mathbb{R}^{N}$,

$$
J_{\delta}(u, x):=\int_{\left\{\left|\Psi_{u}(x, y)-\Psi_{u}(x, x)\right|>\delta\right\}} \frac{\delta^{2}}{|x-y|^{N+2}} \mathrm{~d} y .
$$

We have

Proposition 6.2 ([15, Proposition 4.2]): Let $A: \mathbb{R}^{N} \rightarrow \mathbb{R}^{N}$ be Lipschitz and let $u \in$ $H_{A}^{1}\left(\mathbb{R}^{N}\right)$. Then

$$
\lim _{\delta \searrow 0} J_{\delta}(u, x)=Q_{N}|\nabla u(x)-\mathrm{i} A(x) u(x)|^{2}, \quad \text { for a.e. } x \in \mathbb{R}^{N}
$$

and

$$
\lim _{\delta \searrow 0} J_{\delta}(u, \cdot)=Q_{N}|\nabla u(\cdot)-\mathrm{i} A(\cdot) u(\cdot)|^{2}, \quad \text { in } L^{1}\left(\mathbb{R}^{N}\right)
$$

In both cases, we prove the results on smooth functions relying on delicate estimates of maximal-type functions with their roots in [4]. We can then conclude arguing by density. We refer to [15] for detailed proofs of both results.

\section{Note}

1. Some of these works only deal with the whole space setting, nevertheless, one can extend them for a smooth bounded domains as stated. 


\section{Acknowledgements}

A.P., M.S. and E.V. are members of Gruppo Nazionale per l'Analisi Matematica, la Probabilità e le loro Applicazioni (GNAMPA) of the Istituto Nazionale di Alta Matematica (INdAM).

\section{Disclosure statement}

No potential conflict of interest was reported by the authors.

\section{References}

[1] Bourgain J, Brézis H, Mironescu P. Another look at Sobolev spaces. In Menaldi JL, Rofman E, and Sulem A, editors. Optimal control and partial differential equations. A volume in honor of Professor Alain Bensoussan's 60th birthday. Amsterdam: IOS Press; 2001. p. 439-455.

[2] Davila J. On an open question about functions of bounded variation. Calc Var Partial Differ Equ. 2002;15:519-527.

[3] Brézis H. New approximations of the total variation and filters in imaging. Rend Accad Lincei. 2015;26:223-240.

[4] Brézis H, Nguyen H-M. The BBM formula revisited. Atti Accad Naz Lincei Rend Lincei Mat Appl. 2016;27:515-533.

[5] Brézis H, Nguyen H-M. Two subtle convex nonlocal approximations of the BV-norm. Nonlinear Anal. 2016;137:222-245.

[6] Maz'ya V, Shaposhnikova T. On the Bourgain, Brezis, and Mironescu theorem concerning limiting embeddings of fractional Sobolev spaces. J Funct Anal. 2002;195:230-238. Erratum to: On the Bourgain, Brezis and Mironescu theorem concerning limiting embeddings of fractional Sobolev spaces. J Funct Anal. 2003;201:298-300.

[7] Bourgain J, Nguyen H-M. A new characterization of Sobolev spaces. C R Acad Sci Paris. 2006;343:75-80.

[8] Nguyen H-M. Some new characterizations of Sobolev spaces. J Funct Anal. 2006;237:689-720.

[9] Brézis H, Nguyen H-M. Non-local functionals related to the total variation and connections with image processing. Ann PDE. 2018;4:9. Available from: https://doi.org/10.1007/s40818018-0044-1

[10] Nguyen H-M. Г-convergence, Sobolev norms, and BV functions. Duke Math J. 2011;157: 495-533.

[11] Nguyen H-M. Some inequalities related to Sobolev norms. Calc Var Partial Differ Equ. 2011;41:483-509.

[12] Nguyen H-M. Estimates for the topological degree and related topics. J Fixed Point Theory. 2014;15:185-215.

[13] Nguyen H-M, Squassina M. Some remarks on rearrangement for nonlocal functionals. Nonlinear Anal. 2017;162:1-12.

[14] Nguyen H-M, Squassina M. On anisotropic Sobolev spaces. Commun Contemp Math, to appear. DOI:10.1142/S0219199718500177

[15] Nguyen H-M, Pinamonti A, Squassina M, et al. New characterizations of magnetic Sobolev spaces. Adv Nonlinear Anal. 2018;7(2):227-245.

[16] Pinamonti A, Squassina M, Vecchi E. Magnetic BV functions and the Bourgain-BrezisMironescu formula. Adv Calc Var, to appear. DOI:10.1515/acv-2017-0019

[17] Pinamonti A, Squassina M, Vecchi E. The Maz'ya-Shaposhnikova limit in the magnetic setting. J Math Anal Appl. 2017;449:1152-1159.

[18] Squassina M, Volzone B. Bourgain-Brezis-Mironescu formula for magnetic operators. C R Math Acad Sci Paris. 2016;354:825-831.

[19] Avron J, Herbst I, Simon B. Schrödinger operators with magnetic fields. I. General interactions. Duke Math J. 1978;45:847-883.

[20] Reed M, Simon B. Methods of modern mathematical physics, I, functional analysis. New York: Academic Press, Inc.; 1980. 
[21] Arioli G, Szulkin A. A semilinear Schrödinger equation in the presence of a magnetic field. Arch Ration Mech Anal. 2003;170:277-295.

[22] Esteban M, Lions P-L. Stationary solutions of nonlinear Schrödinger equations with an external magnetic field. In Partial differential equations and the calculus of variations. Vol. I. Boston (MA): Birkhäuser Boston; 1989. p. 401-449. (Progr. nonlinear differential equations appl.; 1).

[23] Squassina M. Soliton dynamics for the nonlinear Schrödinger equation with magnetic field. Manuscripta Math. 2009;130:461-494.

[24] Lieb EH, Loss M. Analysis. Providence (RI): American Mathematical Society; 2001. (Graduate studies in mathematics; 14).

[25] Di Nezza E, Palatucci G, Valdinoci E. Hitchhiker's guide to the fractional Sobolev spaces. Bull Sci Math. 2012;136:521-573.

[26] Ichinose T. Magnetic relativistic Schrödinger operators and imaginary-time path integrals. In: Mathematical physics, spectral theory and stochastic analysis. Basel: Birkhäuser/Springer; 2013. p. 247-297. (Oper. theory adv. appl.; 232).

[27] Binlin Z, Squassina M, Xia Z. Fractional NLS equations with magnetic field, critical frequency and critical growth. Manuscripta Math. 2018;155(1-2):115-140.

[28] d'Avenia P, Squassina M. Ground states for fractional magnetic operators. ESAIM COCV. 2018;24(1):1-24.

[29] Fiscella A, Pinamonti A, Vecchi E. Multiplicity results for magnetic fractional problems. J Differ Equ. 2017;263(8):4617-4633.

[30] Liang S, Repovs D, Zhang B. On the fractional Schrödinger-Kirchhoff equations with electromagnetic fields and critical nonlinearity. Comput Math Appl. 2018;75:1778-1794.

[31] Mingqi X, Pucci P, Squassina M, et al. Nonlocal Schrödinger-Kirchhoff equations with external magnetic field. Discrete Contin Dyn Syst A. 2017;37:503-521.

[32] Mingqi X, Radulescu V, B. Zhang V. A critical fractional Choquard-Kirchhoff problem with magnetic field. Commun Contemp Math. 2018, to appear. DOI:10.1142/S0219199718500049

[33] Wang F, Xiang M. Multiplicity of solutions to a nonlocal Choquard equation involving fractional magnetic operators and critical exponent. Electron J Differ Equ. 2016;2016:1-11.

[34] Ambrosio L, Fusco N, Pallara D. Functions of bounded variation and free discontinuity problems. New York: The Clarendon Press, Oxford University Press; 2000.

[35] Brézis H. How to recognize constant functions. Connections with Sobolev spaces. Russian Math Surveys. 2002;57:693-708.

[36] Bourgain J, Brézis H, Mironescu P. Limiting embedding theorems for $W^{s, p}$ when $s \uparrow 1$ and applications. J Anal Math. 2002;87:77-101.

[37] Ponce A. A new approach to Sobolev spaces and connections to $\Gamma$-convergence. Calc Var Partial Differ Equ. 2004;19:229-255.

[38] Ponce A, Spector D. On formulae decoupling the total variation of BV functions. Nonlinear Anal. 2017;154:241-257. 